\title{
Feasibility Study of Aseptic Homogenization: Affecting Homogenization Steps on Quality of Sterilized Coconut Milk
}

\author{
Chanthima Phungamngoen ${ }^{1, a}$, Tippunsa Asawajinda ${ }^{1}$, Rujira Santad ${ }^{1}$, Wanticha Sawedboworn $^{1}$ \\ ${ }^{1}$ King Mongkut's University of Technology North Bangkok, 129 M. 21 Noenhom, Maung, Prachinburi, 25230 Thailand
}

\begin{abstract}
Coconut milk is one of the most important protein-rich food sources available today. Separation of an emulsion into an aqueous phase and cream phase is commonly occurred and this leads an unacceptably physical defect of either fresh or processed coconut milk. Since homogenization steps are known to affect the stability of coconut milk. This work was aimed to study the effect of homogenization steps on quality of coconut milk. The samples were subject to high speed homogenization in the range of 5000-15000 rpm under sterilize temperatures at $120-140{ }^{\circ} \mathrm{C}$ for $15 \mathrm{~min}$. The result showed that emulsion stability increase with increasing speed of homogenization. The lower fat particles were generated and easy to disperse in continuous phase lead to high stability. On the other hand, the stability of coconut milk decreased, fat globule increased, $L$ value decreased and $b$ value increased when the high sterilization temperature was applied. Homogenization after heating led to higher stability than homogenization before heating due to the reduced particle size of coconut milk after aggregation during sterilization process. The results implied that homogenization after sterilization process might play an important role on the quality of the sterilized coconut milk.
\end{abstract}

\section{Introduction}

Coconut milk is a liquid produced by soaking the white inner flesh of the seed of the coconut in water and straining. Coconut milk is commonly used in South East Asian cuisine for its aromatic property in curry and other gravy. The major components of coconut milk are water and fat which fat globules disperse throughout the water phase [1].

Sterilized coconut milk required high temperature for long processing time because it is an emulsion and low acid food ( $\mathrm{pH} 7$ ). Separation of an emulsion into an aqueous phase and cream phase is commonly occurred and this leads an unacceptably physical defect of either fresh or processed coconut milk [2]. Moreover, the protein denaturation occurred leading to curding in liquid system occured when temperature reaching to $80{ }^{\circ} \mathrm{C}$ [3] which can affect the stability of the milk. Previous research works have demonstrated that fat particle size, dispersion, homogenization and temperature had significant effects on a stability of coconut milk $[2,4,5]$. However, after heated coconut milk product with high temperature leads to much quality degradation, i.e., separation and brown color in product. In the sterilization process of coconut milk, there is no homogenization again therefore had the opportunity to make the product less stable.

Since homogenization steps are known to affect the stability of coconut milk, it is interesting to study the effects of homogenization steps on the stability, fat particle size and color of sterilized coconut milk. In this study, attempt was made to feasibility study of aseptic homogenization. The opjective of this work was to study the effect of homogenization steps on somes quality of coconut milk.

\section{Materials and Methods}

\subsection{Sample preparation}

Coconut milk without added water from a local market was used in the experiments. The initial fat content of coconut milk was determined using Rose-Gottlieb method [6]. The fresh coconut milk was diluted by distilled water to obtain the coconut milk with $20 \% \mathrm{w} / \mathrm{v}$ fat. Emulsifier stabilizer mixture of Montanox 60 (Adinop, Thailand) and CMC (Thai Food and Chemical, Thailand) with a ratio of $0.6: 0.6 \% \mathrm{w} / \mathrm{v}$ [3] were added while the samples were heated and stirred continuously until sample temperature reached $70^{\circ} \mathrm{C}$.

\subsection{Homogenization steps}

\subsubsection{Effect of homogenization speed and heating temperature}

Applied the single step of homogenization (Polytron, model PT-MR 3100, Germany) at speeds of 5,000,

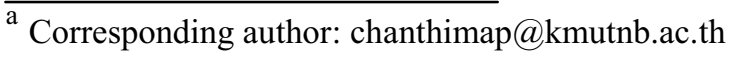


10,000 and $15,000 \mathrm{rev} / \mathrm{min}$ for 2 minutes ( $1^{\text {st }}$ steps). Then heat sample in oil bath at a temperature of 120, 130 and $140{ }^{\circ} \mathrm{C}$ for 15 minutes.

\subsubsection{Effect of homogenization steps}

Applied the two steps of homogenization at speeds of $5,000,10,000$ and $15,000 \mathrm{rev} / \mathrm{min}$ for 2 minutes $\left(1^{\text {st }} \mathrm{step}\right)$ and heating sample at 120,130 and $140{ }^{\circ} \mathrm{C}$ for 15 minutes after that homogenization again at speeds of 500 , 1,000 and 5,000 rev/min for 1 minutes ( $2^{\text {nd }}$ step $)$.

\subsection{Determine quality of coconut milk}

\subsubsection{Emulsion stability (\%ES)}

To evaluate the stability, the sterilized samples were kept at room temperature $\left(\sim 30^{\circ} \mathrm{C}\right)$ for 3 days and determined the stability of emulsion following the method described by Phungamngoen and others [4]. The emulsion stability can be calculated by following formula:

$$
\% \mathrm{ES}=\frac{\text { Height of cream phase }}{\text { Height of whole coconut milk }} \times 100
$$

\subsubsection{Droplet size}

A few drops of oleoresin dye were added to $10 \mathrm{~mL}$ coconut milk sample which was stirred for at least $1 \mathrm{~min}$ to disperse the dye. A few drops of the sample were transformed to the slide and a cover slip was placed over the sample. An optical standard microscope (Olympus Model CH30, Japan) was used to determine the fat structure at a magnification of $400 \times$ and photographs were taken from typical fields.

\subsubsection{Color}

Color of samples was measured in a Hunter colorimeter (Hunter Lab, Model Colorflex 45/0, Virginia) Reflection spectra were registered and Hunter Lab color parameters for $10^{\circ}$ vision angle and D65 illuminant ( $L$ : brigthness, $a$ : redness, $b$ : yellowness) were calculated. Measurements were done in triplicate on each of two subsamples.

\subsection{Statistical analysis}

All data were analyzed using the analysis of variance (ANOVA) and were presented as mean values with standard deviations. Differences between mean values were established using Duncan's new multiple range test; values were considered at a confidence level of $95 \%$. SPSS (version 10.0 statistics software, SPSS Inc., Chicago, IL) was used to perform all statistical calculations.

\section{Results and Discussion}

\subsection{Effect on emulsion stability}

Table 1 shows emulsion stability (\% ES) of coconut milk as affect of homogenizing steps and sterilizing temperature. The result showed that the non homogenized sample had lower stability than homogenized ones. The $\%$ ES value of coconut milks increased as the homogenization speed increased. Increaseing heating temperature exhibited low stabity of product. This observation was consistent with a previous work which studied the effect of homogenizing pressure and sterilizing condition on quality of canned high fat coconut milk [2].

Homogenization after heating (2 steps homogenization) leading to higher \% ES at the same hogenization speed when compare with hogenization only one step. It was observed that increasing $2^{\text {nd }}$ step of homogenization speed did not have significant effect on $\% \mathrm{ES}$ of coconut milk.

Table 1. Effect of homogenizing steps and sterilizing temperature on emulsion stability (\%ES) of coconut milk.

\begin{tabular}{|c|c|c|c|}
\hline \multirow{2}{*}{$\begin{array}{c}\mid 3 \\
\text { Homogenization }\end{array}$} & \multicolumn{3}{|c|}{ Temperature } \\
\cline { 2 - 4 } steps & $120^{\circ} \mathrm{C}$ & $130^{\circ} \mathrm{C}$ & $140^{\circ} \mathrm{C}$ \\
\hline Non-homogenized & $70.12^{\mathrm{e}}$ & $68.33^{\mathrm{e}}$ & $66.41^{\mathrm{e}}$ \\
sample (control) & $(3.01)$ & $(1.97)$ & $(4.20)$ \\
\hline $5,000 \mathrm{rev} / \mathrm{min}$ & $78.85^{\mathrm{cde}}$ & $78.31^{\mathrm{cde}}$ & $78.23^{\mathrm{cde}}$ \\
& $(5.77)$ & $(6.31)$ & $(1.31)$ \\
\hline $10,000 \mathrm{rev} / \mathrm{min}$ & $82.31^{\mathrm{cd}}$ & $81.92^{\mathrm{cd}}$ & $79.20^{\mathrm{cde}}$ \\
& $(2.31)$ & $(2.69)$ & $(2.28)$ \\
\hline $15,000 \mathrm{rev} / \mathrm{min}$ & $82.28^{\mathrm{cd}}$ & $83.86^{\mathrm{cd}}$ & $80.77^{\mathrm{bc}}$ \\
& $(4.80)$ & $(0.14)$ & $(3.85)$ \\
\hline $5,000 \mathrm{rev} / \mathrm{min}-H e a t-$ & $85.54^{\mathrm{bcd}}$ & $82.92^{\mathrm{cd}}$ & $78.85^{\mathrm{cd}}$ \\
$500 \mathrm{rev} / \mathrm{min}$ & $(2.46)$ & $(3.00)$ & $(1.92)$ \\
\hline $5,000 \mathrm{rev} / \mathrm{min}-H e a t-$ & $86.31^{\mathrm{bc}}$ & $83.34^{\mathrm{cd}}$ & $77.69^{\mathrm{cd}}$ \\
$1000 \mathrm{rev} / \mathrm{min}$ & $(1.69)$ & $(2.35)$ & $(0.77)$ \\
\hline $5,000 \mathrm{rev} / \mathrm{min}-H e a t-$ & $86.54^{\mathrm{bc}}$ & $82.57^{\mathrm{cd}}$ & $79.43^{\mathrm{cd}}$ \\
$5000 \mathrm{rev} / \mathrm{min}$ & $(1.92)$ & $(4.11)$ & $(0.73)$ \\
\hline $10,000 \mathrm{rev} / \mathrm{min}-H e a t-$ & $87.98^{\mathrm{bc}}$ & $89.43^{\mathrm{ab}}$ & $90.38^{\mathrm{ab}}$ \\
$500 \mathrm{rev} / \mathrm{min}$ & $(0.56)$ & $(1.54)$ & $(1.92)$ \\
\hline $10,000 \mathrm{rev} / \mathrm{min}-H e a t-$ & $89.75^{\mathrm{ab}}$ & $89.77^{\mathrm{ab}}$ & $90.00^{\mathrm{b}}$ \\
$1000 \mathrm{rev} / \mathrm{min}$ & $(2.25)$ & $(3.14)$ & $(0.40)$ \\
\hline $10,000 \mathrm{rev} / \mathrm{min}-H e a t-$ & $88.14^{\mathrm{b}}$ & $89.47^{\mathrm{ab}}$ & $90.79^{\mathrm{ab}}$ \\
$5000 \mathrm{rev} / \mathrm{min}$ & $(3.53)$ & $(2.81)$ & $(2.22)$ \\
\hline $15,000 \mathrm{rev} / \mathrm{min}-H e a t-$ & $92.11^{\mathrm{a}}$ & $91.01^{\mathrm{ab}}$ & $90.37^{\mathrm{ab}}$ \\
$500 \mathrm{rev} / \mathrm{min}$ & $(1.13)$ & $(0.10)$ & $(4.92)$ \\
\hline $15,000 \mathrm{rev} / \mathrm{min}-H e a t-$ & $92.25^{\mathrm{a}}$ & $91.99^{\mathrm{ab}}$ & $90.84^{\mathrm{ab}}$ \\
$1000 \mathrm{rev} / \mathrm{min}$ & $(1.48)$ & $(1.08)$ & $(3.55)$ \\
\hline $15,000 \mathrm{rev} / \mathrm{min}-H e a t-$ & $92.00^{\mathrm{a}}$ & $90.80^{\mathrm{ab}}$ & $90.51^{\mathrm{ab}}$ \\
$5000 \mathrm{rev} / \mathrm{min}$ & $(0.33)$ & $(2.00)$ & $(2.97)$ \\
\hline
\end{tabular}

Values in the same column with different superscripts mean that the values are significantly different $(\mathrm{p}<0.05)$.

\subsection{Effect on fat particle size}

The effect of homogenization speed on fat particle size of coconut milk were conducted using optical standard microscope (Figure 1-4). During the homogenization, the high shear forces acted on dispersed phase to reduce droplet size [7]. Small fat globule sizes were obtained at higher homogenization speed. Reduction in the fat 
particle diameters resulted in an increase in $\% \mathrm{ES}$ value. When the homogenized coconut milk samples were subjected to heat treatments, small fat globules formed irregular rearrangement of aggregates (Figure 2). Naturally, coconut milk composes of fat globules surrounded by the aqueous protein solution $[2,8,9]$.

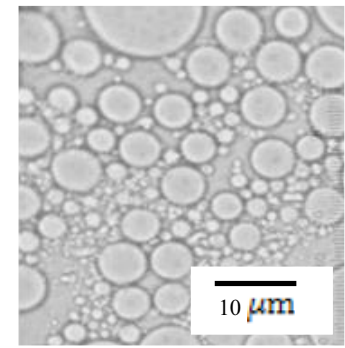

Figure 1. Micrographs $(\times 400$ magnification $)$ of fresh coconut milk sample.

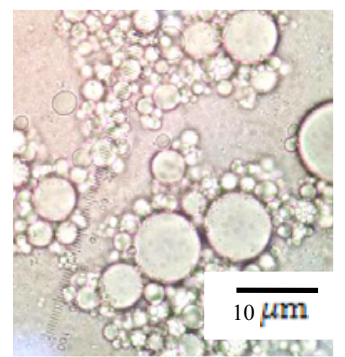

(a)

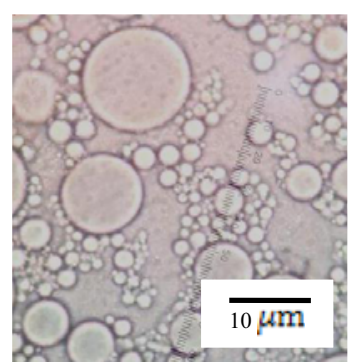

(b)

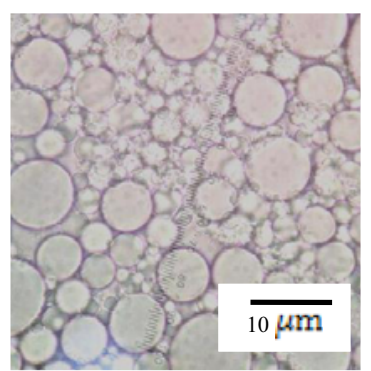

(c)

Figure 2. Micrographs $(\times 400$ magnification $)$ of coconut milk samples passing homogenization at $5000 \mathrm{rev} / \mathrm{min}$ with different heating temperature; (a) $120^{\circ} \mathrm{C}$, (b) $130{ }^{\circ} \mathrm{C}$ and (c) $140{ }^{\circ} \mathrm{C}$.

Figure 3 shows micrographs of coconut milk samples passing different conditions. At the same heating temperature, higher $2^{\text {nd }}$ steps of homogenization speeds resulted in the same size of fat particle size. There is no benefit in increasing stability of coconut milk product when it used 2 steps homogenization. This was due to the ideal second-step homogenization should be between 10 and $15 \%$ of the total homogenization speed. It has been demonstrated with emulsion that passage through the second-stage valve, itself, does not significantly alter the condition of the emulsion. Sampling the emulsion both before and after the second-stage did not reveal any substantial change in emulsion quality $[10,11]$. The temperature differences between the homogenization speed at higher heating temperatures gave larger fat particle size than that at a lower temperature (data not shown).

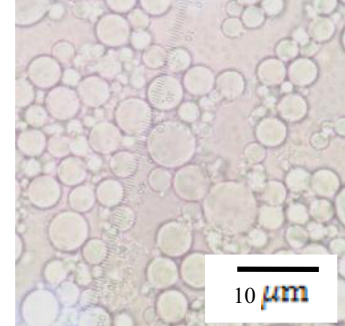

(a)

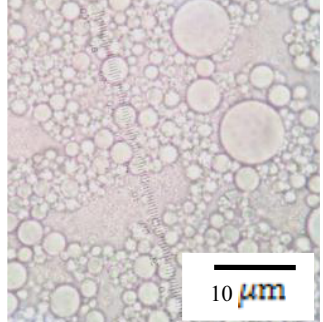

(b)

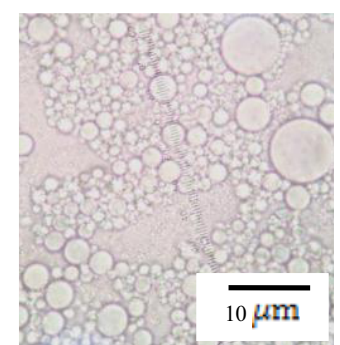

(c)

Figure 3. Micrographs ( $\times 400$ magnification) of coconut milk samples passing different conditions; (a) 5,000 rpm-heat 120 ${ }^{\circ} \mathrm{C}-500 \mathrm{rpm}$, (b) $5,000 \mathrm{rpm}$-heat $120^{\circ} \mathrm{C}-1,000 \mathrm{rpm}$ and (c) $5,000 \mathrm{rpm}$-heat $120^{\circ} \mathrm{C}-5,000 \mathrm{rpm}$.

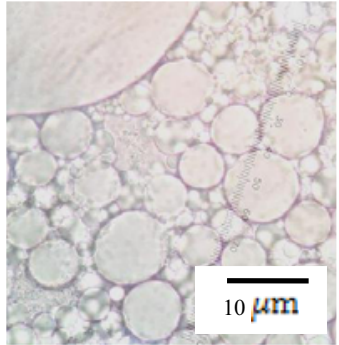

$(\mathrm{a}-1)$

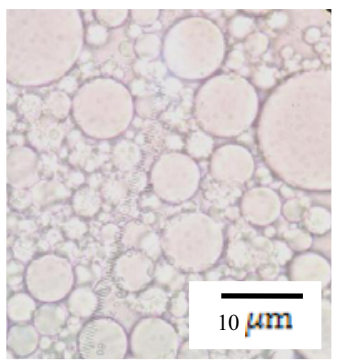

$(\mathrm{a}-2)$

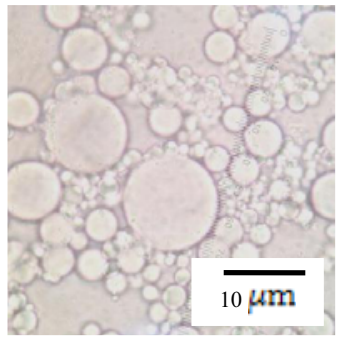

$(\mathrm{a}-3)$

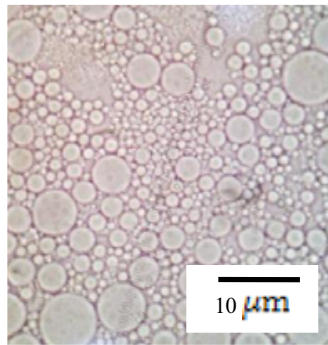

$(b-1)$

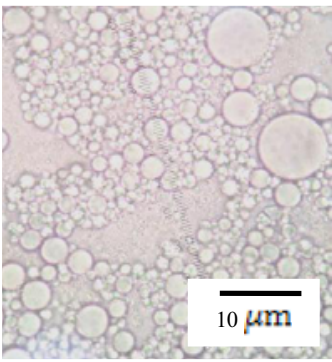

$(\mathrm{b}-2)$

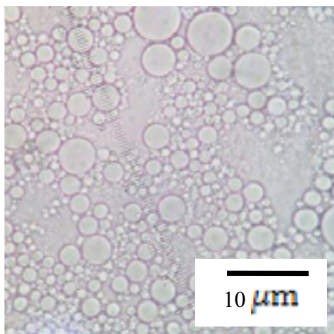

$(b-3)$
Figure 4. Micrographs ( $\times 400$ magnification $)$ of coconut milk samples passing different conditions; homogenized before heating at $120{ }^{\circ} \mathrm{C}(\mathrm{a}-1)$ 5,000 rpm, (a-2) 10,000 rpm, (a-3) $15,000 \mathrm{rpm}$; homogenized before and after heating at $120^{\circ} \mathrm{C}(\mathrm{b}-$ 1) $5,000 \mathrm{rpm}$-heat-500 rpm, (b) $10,000 \mathrm{rpm}$-heat-500 rpm and (c) $15,000 \mathrm{rpm}$-heat $-500 \mathrm{rpm}$. 
Figure 4 shows exemplifies comparing effect of one step (Figure 4: a-1, a-2 and a-3) and two steps of homogenization speed (Figure $4: \mathrm{b}-1, \mathrm{~b}-2$ and b-3). It was observe that two steps of homogenization speed led to smaller fat particle size than one step homogenization speed. This might be due to the fact that during heating the samples with high temperature gained fat globules tended to form aggregates [2]. At this temperature, the protein denaturation occurred leading to curding in liquid system [3]. Therefore, applying homogenization speed after heating was applicable for improving the stability of coconut milk samples. The micrographs supported the results from the emulsion stability studies that increasing in $\% \mathrm{ES}$ of heated treated homogenized coconut milk was caused from the change in microstructure.

\subsection{Effect on color}

It is seen in Table 2-4 that the applied homogenization speed and heating temperature showed significant effect on the color of coconut milk. In general, lowering the speed of homogenization and lower heating temperature helped to prevent browning reaction and color degradation in coconut milk [4]. It was obvious that high homogenization speed resulted in an increase in the $L$ value and a decrease in the $b$ value. However, it was observed in this work that increasing $2^{\text {nd }}$ steps of homogenization speed did not have a significant effect on the color of coconut milk.

Table 2. Effect of homogenizing steps on color of sterilized coconut milk at $120^{\circ} \mathrm{C}$.

\begin{tabular}{|c|c|c|c|}
\hline \multirow{2}{*}{$\begin{array}{l}\text { Homogenization } \\
\text { steps }\end{array}$} & \multicolumn{3}{|c|}{ Color } \\
\hline & $L$ & $a$ & $b$ \\
\hline $\begin{array}{c}\text { Non-homogenized } \\
\text { sample }\end{array}$ & $\begin{array}{l}77.72^{f} \\
(0.03) \\
\end{array}$ & $\begin{array}{l}0.87^{\mathrm{a}} \\
(0.03)\end{array}$ & $\begin{array}{l}7.73^{\mathrm{b}} \\
(0.02) \\
\end{array}$ \\
\hline $5,000 \mathrm{rev} / \mathrm{min}$ & $\begin{array}{l}80.38^{\mathrm{e}} \\
(0.22)\end{array}$ & $\begin{array}{l}0.46^{6} \\
(0.03)\end{array}$ & $\begin{array}{l}7.38^{a} \\
(0.03)\end{array}$ \\
\hline $10,000 \mathrm{rev} / \mathrm{min}$ & $\begin{array}{l}82.52^{\mathrm{d}} \\
(0.33)\end{array}$ & $\begin{array}{l}-0.74^{\mathrm{g}} \\
(0.12)\end{array}$ & $\begin{array}{l}5.59^{b} \\
(0.23)\end{array}$ \\
\hline $15,000 \mathrm{rev} / \mathrm{min}$ & $\begin{array}{l}85.51^{\mathrm{c}} \\
(0.10)\end{array}$ & $\begin{array}{l}0.30^{\text {def }} \\
(0.02)\end{array}$ & $\begin{array}{l}4.93^{\text {cd }} \\
(0.07)\end{array}$ \\
\hline $\begin{array}{l}5,000 \mathrm{rev} / \mathrm{min}-\mathrm{Heat}- \\
500 \mathrm{rev} / \mathrm{min}\end{array}$ & $\begin{array}{l}85.83^{\mathrm{bc}} \\
(0.24)\end{array}$ & $\begin{array}{l}0.23^{\mathrm{f}} \\
(0.02)\end{array}$ & $\begin{array}{l}5.04^{\mathrm{cd}} \\
(0.10)\end{array}$ \\
\hline $\begin{array}{c}5,000 \mathrm{rev} / \mathrm{min}-\mathrm{Heat}- \\
1000 \mathrm{rev} / \mathrm{min}\end{array}$ & $\begin{array}{l}86.66^{\mathrm{b}} \\
(0.06)\end{array}$ & $\begin{array}{l}0.28^{\mathrm{def}} \\
(0.02)\end{array}$ & $\begin{array}{l}5.14^{\text {cd }} \\
(0.01)\end{array}$ \\
\hline $\begin{array}{l}5,000 \mathrm{rev} / \mathrm{min}-\mathrm{Heat}- \\
5000 \mathrm{rev} / \mathrm{min}\end{array}$ & $\begin{array}{c}86.65^{\mathrm{ab}} \\
(0.42)\end{array}$ & $\begin{array}{l}0.33^{\text {cde }} \\
(0.02)\end{array}$ & $\begin{array}{l}5.00^{\text {cd }} \\
(0.17)\end{array}$ \\
\hline $\begin{array}{c}10,000 \mathrm{rev} / \mathrm{min}-\mathrm{Heat}- \\
500 \mathrm{rev} / \mathrm{min}\end{array}$ & $\begin{array}{l}86.40^{b} \\
(0.17)\end{array}$ & $\begin{array}{l}0.34^{\text {cde }} \\
(0.02)\end{array}$ & $\begin{array}{l}4.81^{\text {cde }} \\
(0.14)\end{array}$ \\
\hline $\begin{array}{l}10,000 \mathrm{rev} / \mathrm{min}-\mathrm{Heat}- \\
1000 \mathrm{rev} / \mathrm{min}\end{array}$ & $\begin{array}{l}86.56^{b} \\
(0.35)\end{array}$ & $\begin{array}{l}0.39^{\mathrm{c}} \\
(0.02)\end{array}$ & $\begin{array}{l}4.91^{\text {bc }} \\
(0.14)\end{array}$ \\
\hline $\begin{array}{c}10,000 \mathrm{rev} / \mathrm{min}-\mathrm{Heat}- \\
5000 \mathrm{rev} / \mathrm{min}\end{array}$ & $\begin{array}{l}87.87^{\mathrm{a}} \\
(0.13)\end{array}$ & $\begin{array}{l}0.27^{\text {def }} \\
(0.04)\end{array}$ & $\begin{array}{l}4.59^{\text {cd }} \\
(0.10)\end{array}$ \\
\hline $\begin{array}{c}15,000 \mathrm{rev} / \mathrm{min}-H e a t- \\
500 \mathrm{rev} / \mathrm{min}\end{array}$ & $\begin{array}{l}88.07^{a} \\
(0.04)\end{array}$ & $\begin{array}{l}0.24^{f} \\
(0.03)\end{array}$ & $\begin{array}{l}4.56^{\text {cd }} \\
(0.06)\end{array}$ \\
\hline $\begin{array}{l}15,000 \mathrm{rev} / \mathrm{min}-H e a t- \\
1000 \mathrm{rev} / \mathrm{min}\end{array}$ & $\begin{array}{l}88.36^{\mathrm{a}} \\
(0.22)\end{array}$ & $\begin{array}{l}0.23^{f} \\
(0.03)\end{array}$ & $\begin{array}{l}4.48^{\text {cd }} \\
(0.08)\end{array}$ \\
\hline $\begin{array}{l}15,000 \mathrm{rev} / \mathrm{min}-\mathrm{Heat}- \\
5000 \mathrm{rev} / \mathrm{min}\end{array}$ & $\begin{array}{l}88.23^{\mathrm{a}} \\
(0.31)\end{array}$ & $\begin{array}{l}0.25^{\text {ef }} \\
(0.03)\end{array}$ & $\begin{array}{l}4.72^{\mathrm{c}} \\
(0.07)\end{array}$ \\
\hline
\end{tabular}

Values in the same column with different superscripts mean that the values are significantly different $(\mathrm{p}<0.05)$.
Among the studied two steps homogenization speed had higher $L$ and lower $b$ values compared to the one step homogenization speed. Smaller droplets were produced when the higher homogenization speeds were applied. The reflectance increased with increasing droplet concentration and decreasing droplet size $[12,13]$.

\section{Conclusion}

The effects of homogenization steps (before sterilization process and homogenization before and after sterilization) on the stability, fat particle size, viscosity and color of sterilized coconut milk were investigated. The result showed that emulsion stability increase with increasing speed of homogenization because the higher speed of homogenization. High sterilization temperature led to $L$ value decreased and $b$ value increased. Homogenization after heating gave higher stability than homogenization before heating. The results implied that homogenization after sterilization process might play an important role on the quality of the sterilized coconut milk. The results could be used as a guidance for producing sterilized coconut milk with aseptic homogenizer.

Table 3. Effect of homogenizing steps on color of sterilized coconut milk at $130{ }^{\circ} \mathrm{C}$.

\begin{tabular}{|c|c|c|c|}
\hline \multirow{2}{*}{$\begin{array}{l}\text { Homogenization } \\
\text { steps }\end{array}$} & \multicolumn{3}{|c|}{ Color } \\
\hline & $L$ & $a$ & $b$ \\
\hline $\begin{array}{l}\text { Non-homogenized } \\
\text { sample }\end{array}$ & $\begin{array}{l}75.56^{f} \\
(0.14)\end{array}$ & $\begin{array}{l}1.01^{\mathrm{a}} \\
(0.03)\end{array}$ & $\begin{array}{l}8.16^{\mathrm{a}} \\
(0.02)\end{array}$ \\
\hline $5,000 \mathrm{rev} / \mathrm{min}$ & $\begin{array}{l}78.38^{\mathrm{c}} \\
(0.22)\end{array}$ & $\begin{array}{l}0.46^{\mathrm{b}} \\
(0.03)\end{array}$ & $\begin{array}{l}5.21^{\mathrm{b}} \\
(0.10)\end{array}$ \\
\hline $10,000 \mathrm{rev} / \mathrm{min}$ & $\begin{array}{l}80.60^{b} \\
(0.37)\end{array}$ & $\begin{array}{l}-0.55^{\mathrm{d}} \\
(0.08)\end{array}$ & $\begin{array}{l}5.04^{\text {bc }} \\
(0.07)\end{array}$ \\
\hline $15,000 \mathrm{rev} / \mathrm{min}$ & $\begin{array}{l}82.21^{\mathrm{ab}} \\
(0.18)\end{array}$ & $\begin{array}{l}-0.45^{\mathrm{c}} \\
(0.04)\end{array}$ & $\begin{array}{l}4.93^{\mathrm{cb}} \\
(0.10)\end{array}$ \\
\hline $\begin{array}{l}5,000 \mathrm{rev} / \mathrm{min}-\mathrm{Heat}- \\
500 \mathrm{rev} / \mathrm{min}\end{array}$ & $\begin{array}{l}81.97^{\mathrm{ab}} \\
(0.36)\end{array}$ & $\begin{array}{l}-0.52^{\mathrm{cd}} \\
(0.08)\end{array}$ & $\begin{array}{l}5.14^{\mathrm{b}} \\
(0.01)\end{array}$ \\
\hline $\begin{array}{l}5,000 \mathrm{rev} / \mathrm{min}-\mathrm{Heat}- \\
1000 \mathrm{rev} / \mathrm{min}\end{array}$ & $\begin{array}{l}81.57^{\mathrm{b}} \\
(0.22)\end{array}$ & $\begin{array}{l}-0.58^{\text {de }} \\
(0.05)\end{array}$ & $\begin{array}{l}5.00^{\mathrm{bc}} \\
(0.17)\end{array}$ \\
\hline $\begin{array}{c}5,000 \mathrm{rev} / \mathrm{min}-\mathrm{Heat}- \\
5000 \mathrm{rev} / \mathrm{min}\end{array}$ & $\begin{array}{l}82.90^{\mathrm{a}} \\
(0.07)\end{array}$ & $\begin{array}{l}-0.55^{\mathrm{d}} \\
(0.03)\end{array}$ & $\begin{array}{l}4.81^{\mathrm{c}} \\
(0.14) \\
\end{array}$ \\
\hline $\begin{array}{c}10,000 \mathrm{rev} / \mathrm{min}-\text { Heat- } \\
500 \mathrm{rev} / \mathrm{min}\end{array}$ & $\begin{array}{c}82.23^{\mathrm{ab}} \\
(0.38)\end{array}$ & $\begin{array}{l}-0.57^{\text {de }} \\
(0.09)\end{array}$ & $\begin{array}{l}4.91^{\mathrm{bc}} \\
(0.14)\end{array}$ \\
\hline $\begin{array}{c}10,000 \mathrm{rev} / \mathrm{min}-H e a t- \\
1000 \mathrm{rev} / \mathrm{min}\end{array}$ & $\begin{array}{l}82.84^{\mathrm{a}} \\
(0.31)\end{array}$ & $\begin{array}{l}-0.54^{d} \\
(0.04)\end{array}$ & $\begin{array}{l}4.60^{d} \\
(0.10)\end{array}$ \\
\hline $\begin{array}{c}10,000 \mathrm{rev} / \mathrm{min}-\mathrm{Heat}- \\
5000 \mathrm{rev} / \mathrm{min}\end{array}$ & $\begin{array}{l}83.16^{\mathrm{a}} \\
(0.06)\end{array}$ & $\begin{array}{l}-0.44^{c} \\
(0.03)\end{array}$ & $\begin{array}{l}4.48^{\text {de }} \\
(0.08)\end{array}$ \\
\hline $\begin{array}{c}15,000 \mathrm{rev} / \mathrm{min}-\mathrm{Heat}- \\
500 \mathrm{rev} / \mathrm{min}\end{array}$ & $\begin{array}{l}83.12^{\mathrm{a}} \\
(0.80)\end{array}$ & $\begin{array}{l}-0.59^{\text {de }} \\
(0.09)\end{array}$ & $\begin{array}{l}4.56^{\mathrm{e}} \\
(0.06)\end{array}$ \\
\hline $\begin{array}{c}15,000 \mathrm{rev} / \mathrm{min}-\mathrm{Heat}- \\
1000 \mathrm{rev} / \mathrm{min}\end{array}$ & $\begin{array}{l}83.37^{\mathrm{a}} \\
(0.32) \\
\end{array}$ & $\begin{array}{l}-0.51^{\text {cd }} \\
(0.10)\end{array}$ & $\begin{array}{l}4.72^{\text {cd }} \\
(0.07)\end{array}$ \\
\hline $\begin{array}{c}15,000 \mathrm{rev} / \mathrm{min}-H e a t- \\
5000 \mathrm{rev} / \mathrm{min}\end{array}$ & $\begin{array}{l}83.87^{\mathrm{a}} \\
(0.89)\end{array}$ & $\begin{array}{l}-0.54^{\text {cde }} \\
(0.05)\end{array}$ & $\begin{array}{l}4.67^{\text {cd }} \\
(0.10)\end{array}$ \\
\hline
\end{tabular}

Values in the same column with different superscripts mean that the values are significantly different $(\mathrm{p}<0.05)$. 
Table 4. Effect of homogenizing steps on color of sterilized coconut milk at $140{ }^{\circ} \mathrm{C}$.

\begin{tabular}{|c|c|c|c|}
\hline \multirow{2}{*}{$\begin{array}{l}\text { Homogenization } \\
\text { steps }\end{array}$} & & \multicolumn{2}{|c|}{ Color } \\
\hline & $L$ & $a$ & $b$ \\
\hline $\begin{array}{c}\text { Non-homogenized } \\
\text { sample }\end{array}$ & $\begin{array}{l}72.07^{\mathrm{d}} \\
(0.11)\end{array}$ & $\begin{array}{l}1.08^{\mathrm{a}} \\
(0.03)\end{array}$ & $\begin{array}{l}8.97^{\mathrm{a}} \\
(0.02)\end{array}$ \\
\hline $5,000 \mathrm{rev} / \mathrm{min}$ & $\begin{array}{l}76.36^{\mathrm{c}} \\
(0.07)\end{array}$ & $\begin{array}{l}-0.50^{\text {de }} \\
(0.03)\end{array}$ & $\begin{array}{l}6.45^{\mathrm{b}} \\
(0.03)\end{array}$ \\
\hline $10,000 \mathrm{rev} / \mathrm{min}$ & $\begin{array}{l}80.07^{b} \\
(0.30)\end{array}$ & $\begin{array}{l}-0.44^{\mathrm{d}} \\
(0.08)\end{array}$ & $\begin{array}{l}5.77^{\mathrm{c}} \\
(0.03)\end{array}$ \\
\hline $15,000 \mathrm{rev} / \mathrm{min}$ & $\begin{array}{l}80.81^{\mathrm{ab}} \\
(0.30)\end{array}$ & $\begin{array}{l}-0.51^{\mathrm{d}} \\
(0.05)\end{array}$ & $\begin{array}{l}5.30^{\mathrm{d}} \\
(0.11)\end{array}$ \\
\hline $\begin{array}{l}5,000 \mathrm{rev} / \mathrm{min}-\mathrm{Heat}- \\
500 \mathrm{rev} / \mathrm{min}\end{array}$ & $\begin{array}{l}80.03^{\mathrm{b}} \\
(0.49)\end{array}$ & $\begin{array}{l}-0.50^{\mathrm{de}} \\
(0.08)\end{array}$ & $\begin{array}{l}5.48^{\mathrm{d}} \\
(0.10)\end{array}$ \\
\hline $\begin{array}{c}5,000 \mathrm{rev} / \mathrm{min}-\mathrm{Heat}- \\
1000 \mathrm{rev} / \mathrm{min}\end{array}$ & $\begin{array}{l}80.21^{\mathrm{b}} \\
(0.38)\end{array}$ & $\begin{array}{l}-0.46^{\mathrm{d}} \\
(0.07)\end{array}$ & $\begin{array}{l}5.37^{\mathrm{d}} \\
(0.13)\end{array}$ \\
\hline $\begin{array}{c}5,000 \mathrm{rev} / \mathrm{min}-\mathrm{Heat}- \\
5000 \mathrm{rev} / \mathrm{min}\end{array}$ & $\begin{array}{l}79.26^{b} \\
(0.18)\end{array}$ & $\begin{array}{l}-0.52^{\mathrm{de}} \\
(0.05)\end{array}$ & $\begin{array}{l}5.44^{d} \\
(0.04) \\
\end{array}$ \\
\hline $\begin{array}{c}10,000 \mathrm{rev} / \mathrm{min}-\mathrm{Heat}- \\
500 \mathrm{rev} / \mathrm{min}\end{array}$ & $\begin{array}{l}81.01^{\mathrm{ab}} \\
(0.18)\end{array}$ & $\begin{array}{l}-0.47^{\mathrm{d}} \\
(0.03) \\
\end{array}$ & $\begin{array}{l}5.62^{\mathrm{c}} \\
(0.09) \\
\end{array}$ \\
\hline $\begin{array}{c}10,000 \mathrm{rev} / \mathrm{min}-\mathrm{Heat}- \\
1000 \mathrm{rev} / \mathrm{min}\end{array}$ & $\begin{array}{l}81.53^{\mathrm{a}} \\
(0.34)\end{array}$ & $\begin{array}{l}-0.03^{\mathrm{c}} \\
(0.01)\end{array}$ & $\begin{array}{l}5.58^{\mathrm{c}} \\
(0.06)\end{array}$ \\
\hline $\begin{array}{c}10,000 \mathrm{rev} / \mathrm{min}-H e a t- \\
5000 \mathrm{rev} / \mathrm{min}\end{array}$ & $\begin{array}{l}81.54^{\mathrm{a}} \\
(0.55) \\
\end{array}$ & $\begin{array}{l}-0.02^{\mathrm{c}} \\
(0.05) \\
\end{array}$ & $\begin{array}{l}5.23^{\mathrm{d}} \\
(0.02) \\
\end{array}$ \\
\hline $\begin{array}{c}15,000 \mathrm{rev} / \mathrm{min}-\mathrm{Heat}- \\
500 \mathrm{rev} / \mathrm{min}\end{array}$ & $\begin{array}{l}81.35^{\mathrm{a}} \\
(0.13) \\
\end{array}$ & $\begin{array}{l}0.05^{\mathrm{b}} \\
(0.07)\end{array}$ & $\begin{array}{l}5.25^{d} \\
(0.04) \\
\end{array}$ \\
\hline $\begin{array}{c}15,000 \mathrm{rev} / \mathrm{min}-\mathrm{Heat}- \\
1000 \mathrm{rev} / \mathrm{min}\end{array}$ & $\begin{array}{l}81.23^{\mathrm{a}} \\
(0.17) \\
\end{array}$ & $\begin{array}{c}0.06^{b} \\
(0.04)\end{array}$ & $\begin{array}{l}5.26^{\mathrm{d}} \\
(0.03)\end{array}$ \\
\hline $\begin{array}{c}15,000 \mathrm{rev} / \mathrm{min}-\mathrm{Heat}- \\
5000 \mathrm{rev} / \mathrm{min}\end{array}$ & $\begin{array}{l}81.36^{\mathrm{a}} \\
(0.07) \\
\end{array}$ & $\begin{array}{l}-0.50^{\mathrm{d}} \\
(0.03)\end{array}$ & $\begin{array}{l}5.21^{\mathrm{e}} \\
(0.06) \\
\end{array}$ \\
\hline
\end{tabular}

Values in the same column with different superscripts mean that the values are significantly different $(\mathrm{p}<0.05)$.

\section{Acknowledgements}

The authors express their sincere appreciation to the King Mongkut's University of Technology North Bangkok for financially supporting the study in the form of the General Researcher Grant. Contract no. KMUTNB-GEN57-39.

\section{References}

1. S.P. Ng, O.M. Lai, F. Abas, H.K. Lim, C.P. Tan, Food. Res. Int. 64, 919-930 (2014)

2. N. Chiewchan, C. Phungamngoen, S. Siriwattanayothin, J. Food Eng. 73(1), 38-44 (2006)

3. N. Tangsuphoom, J.N. Coupland, Food Hydrocoll. 23, 1792-1800 (2009)

4. S.N. Raghavendra, K.S.M.S. Raghavarao, J Food Eng. 94, 341-347 (2010)

5. K. Jirapeangtong, S. Siriwatanayothin, N. Chiewchan, J. Food Eng. 87(3), 422-427 (2008)

6. Association of official analytical chemistry (AOAC). Official method of analysis. $15^{\text {th }}$ ed. The association of official agricultural chemists. Virginia, (1990).

7. Z.Y. Yu, S.W. Jiang, X.M. Cao, S.T. Jiang, L.J. Pan, J. Food Eng. 177, 1-8 (2016).

8. T. Peamprasart, N. Chiewchan, J. Food Eng. 77, 653658 (2006)

9. P.R. Borwankar, A. Gonsalves, T.D. Wasan, A. Nikolov, W. Xu, J. Food Sci. 63, 183-188 (1998)

10. N. Tangsuphoom, J.N. Coupland, J. Food Sci. 70, E466-E470 (2005)

11. P. Dhankhar, Int. Org. Sci. Res. J.Eng. 54, 2278 8719 (2014)

12. D.J. McClements, Food emulsions: Principles, practice, and techniques. CRC Press LLC, 161-265 (2005)

13. W. Chantrapornchai, F. Clydesdale, D.J. McClements, Colloids and Surfaces A: Physicochemical and Engineering Aspects, 155, 373382 (1999) 